\title{
Processing Time Reduction: an Application in Living Human High-Resolution Diffusion Magnetic Resonance Imaging Data
}

\author{
Nicolás F . Lori ${ }^{1,2,3,4,5}$ • Augustin Ibañez ${ }^{5,6,7,8,9} \cdot$ Rui Lavrador $^{3}$ - Lucia Fonseca ${ }^{10,11,12}$. \\ Carlos Santos $^{3} \cdot$ Rui Travasso $^{3,10}$ - Artur Pereira ${ }^{13}$ - Rosaldo Rossetti ${ }^{14} \cdot$ Nuno Sousa $^{15}$. \\ Victor Alves ${ }^{1}$
}

Received: 31 May 2016 / Accepted: 5 September 2016

(C) Springer Science+Business Media New York 2016

\begin{abstract}
High Angular Resolution Diffusion Imaging (HARDI) is a type of brain imaging that collects a very large amount of data, and if many subjects are considered then it amounts to a big data framework (e.g., the human connectome project has 20 Terabytes of data). HARDI is also becoming increasingly relevant for clinical settings (e.g.,
\end{abstract}

This article is part of the Topical Collection on Systems-Level Quality Improvement

Nicolás F . Lori

Nicolas.Lori@algoritmi.uminho.pt

Augustin Ibañez

aibanez@ineco.org.ar

Rui Lavrador

rui.lavrador@fmed.uc.pt

Lucia Fonseca

L.T.Neto.Fonseca@tue.nl

Carlos Santos

uc2005107121@student.fis.uc.pt

Rui Travasso

rui@teor.fis.uc.pt

Artur Pereira

artur@ua.pt

Rosaldo Rossetti

rossetti@fe.up.pt

Nuno Sousa

njcsousa@ecsaude.uminho.pt

Victor Alves

Victor.Alves@algoritmi.uminho.pt

1 Algoritmi Centre, University of Minho, Braga, Portugal detecting early cerebral ischemic changes in acute stroke, and in pre-clinical assessment of white matter-WM anatomy using tractography). Thus, this method is becoming a routine assessment in clinical settings. In such settings, the computation time is critical, and finding forms of reducing the processing time in high computation processes such as Diffusion

2 Laboratory of Neuroimaging and Neuroscience (LANEN), Institute of Translational and Cognitive Neuroscience (INCyT), INECO Foundation Rosario, Favaloro University, Rosario, Argentina

3 Institute for Biomedical Imaging and Life Sciences (IBILI), Faculty of Medicine, University of Coimbra, Coimbra, Portugal

4 INECO Neurociencias Oroño, Grupo Oroño, Rosario, Argentina

5 Laboratory of Experimental Psychology and Neuroscience (LPEN), Institute of Translational and Cognitive Neuroscience (INCyT), INECO Foundation, Favaloro University, Buenos Aires, Argentina

6 National Scientific and Technical Research Council (CONICET), Buenos Aires, Argentina

7 Universidad Autónoma del Caribe, Barranquilla, Colombia

8 Department of Psychology, Universidad Adolfo Ibáñez, Santiago, Chile

9 Centre of Excellence in Cognition and its Disorders, Australian Research Council (ACR), Sydney, Australia

10 Center for Physics Computation (CFC), Faculty of Science and Technology, University of Coimbra, Coimbra, Portugal

11 Maastricht University, Maastricht, Netherlands

12 Eindhoven University of Technology, Eindhoven, Netherlands

13 IETTA, University of Aveiro, Aveiro, Portugal

14 LIAC, University of Porto, Porto, Portugal

15 3B's, University of Minho, Braga, Portugal 
Spectrum Imaging (DSI), a form of HARDI data, is very relevant to increase data-processing speed. Here we analyze a method for reducing the computation time of the dMRI-based axonal orientation distribution function $h$ by using Monte Carlo sampling-based methods for voxel selection. Results evidenced a robust reduction in required data sampling of about $50 \%$ without losing signal's quality. Moreover, we show that the convergence to the correct value in this type of Monte Carlo HARDI/DSI data-processing has a linear improvement in data-processing speed of the ODF determination. Although further improvements are needed, our results represent a promissory step for future processing time reduction in big data.

Keywords White matter · Diffusion MRI · Monte Carlo sampling methods $\cdot$ Optimization $\cdot$ Axonal ODF

\section{Introduction}

The brain is one of the most complex structures in the universe given that each neuron is connected with hundreds or thousands of other neurons, not only in the vicinity but also in large-scale networks [1]. Structural connections provide crucial information for healthy, psychiatric and neurological signatures of brain organization [2-5]. Recent technological developments have allowed for big data connectome resourses, open science sharing of large amounts of scans, and massive clinical assessment [1, 6, 7]. Nevertheless, the processing speed for these analyses requirements have been challenged $[1,7]$, increasing computational power and time. Urgent timereduction methods without loss of quality are required for practical use [1, 6-8], especially for multicenter studies and clinical settings.

The goal of this work is to establish how much the use of the Monte Carlo method can increase the speed of complex and large numerical data processing. Specifically, we will consider the determination of the white matter (WM) neural circuitry in humans by the diffusion magnetic resonance imaging (dMRI) data processing, which is often done using the diffusion tensor imaging (DTI) approach [9, 10]. Despite the development of improved dMRI WM fiber tracking techniques [11-15], results are still behind those obtained using in-vitro data [16]; or in-vitro microscopic studies [17]. Thus, to improve WM anatomical representation more gradient orientations and higher gradient intensities have been used which have steadily increased the dMRI's data size and dataprocessing complexity.

The DTI approach represents the diffusion of water molecules in WM by a $3 \times 3$ symmetric matrix, the diffusion tensor, which completely represents that diffusion if the WM is constituted by straight WM fibers all pointing in the same orientation. In this case, the ellipsoid represented by the diffusion tensor has its biggest axis pointing in the orientation of the WM fibers. While for DTI, 6 orientations all with the same gradient amplitude are robust enough, DSI requires hundreds of different gradient orientations and amplitudes.

Different WM fiber tracking techniques have different capacities to accurately represent the anisotropic component of the axonal orientation distribution function (ODF). The bigger the amount of different diffusion-sensitizing parameters the data acquisition has, the more information can be obtained [18-21], but this increases the size of the data set and the corresponding data processing time. A typical example of advanced dMRI data acquisition and processing is Diffusion Spectrum Imaging (DSI) [20]; while for DTI it suffices to have 6 orientations all with the same gradient amplitude, DSI requires hundreds of different gradient orientations and amplitudes. The end-result of the advanced dMRI data processing is often tractography, which consists on showing the WM connections more likely to exist. The final images of the tractography are typically a spaghetti of lines, Fig. 1(a) (e.g. Ref. [12]); and/or a colored anatomical image of connection probabilities, Fig. 1(b) (e.g. Ref. [13]).

We used here the dMRI data processing for extracting the WM axonal ODF, $h$, with an improved estimation of its isotropic and anisotropic fractions. We then calculate how much the use of the Monte Carlo method can reduce the data size required to be within 1 standard error of mean (SEM) from the value obtained using the full data set.

\section{Methods}

\section{Theoretical considerations}

To obtain the axonal ODF, $h$, it is necessary that the experimental dMRI signal is modeled by axons having a certain orientation, and those axons need to have physiologically reasonable properties so that the recovered axonal ODF accurately represents the physiological ODF. Using our model, we determine the parameters which give the best fit of the calculated theoretical dMRI signal to the experimental signal. From the best fit we obtain the experimental axonal ODF, $h_{f i t}$. The experimental axonal ODF can then be separated into an isotropic and an anisotropic component. We proceed to calculate for each voxel the isotropic fraction and the integer $k$ number of WM axes. Then, the number of WM axes in each voxel that are parallel to the WM axes of neighboring voxels is determined by a procedure we developed, which calculates in automatic fashion the WM axes obtained in ref. [15]. Comparing these axis numbers between neighboring voxels, we can quantify the existent number of equally oriented axes in neighboring voxels, we call it framography. This allows us to distinguish between 1-axis connections $(k=1)$, which are lines identical to those obtained in DTI tractography; 2-axes 
Fig. 1 Examples of human brain dMRI data processing, shown are sagital slices of tractography final images. a DTI-based spaghetti of lines, b DTI-based connection probabilities with seed region in corpus callosum
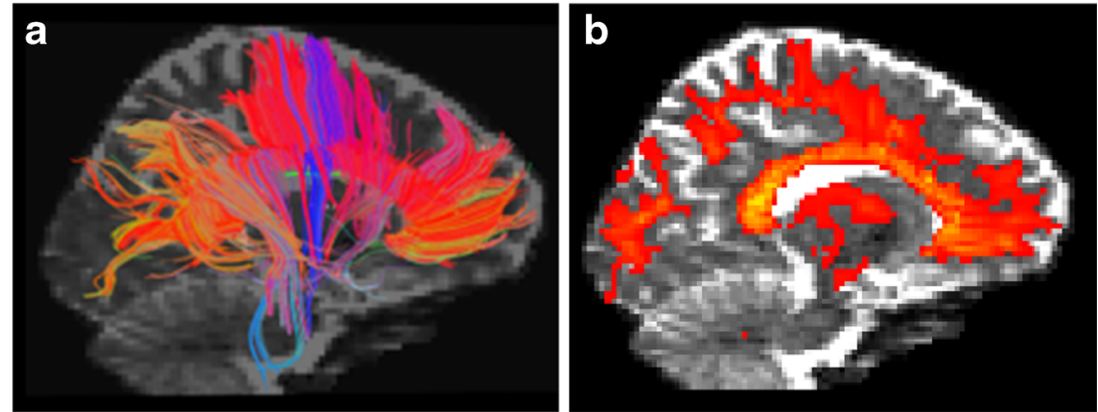

connections $(k=2)$, which occur when 2 fiber-axes at a voxel are compatible with 2 fiber-axes at a neighboring voxel; and 3 axes connections $(k=3)$, which occur when 3 fiber-axes at a voxel are compatible with 3 fiber-axes at a neighboring voxel, similar to ref. [15] (see Fig. 2).

\section{Mathematical representation of dMRI signal}

Using the multi-exponential representation of the dMRI signal in ref. [22], a parameter search is done (see 2.4 sub-section) where $D_{F}$ denotes the diffusion tensor of apparent fast diffusion and $D_{S}$ denotes the diffusion tensor of the apparent slow diffusion orthogonal to the direction of fast diffusion. If the highest eigenvalue of the diffusion tensor for a single axon is $\lambda_{\|}$, and the two smaller eigenvalues are both equal to $\lambda_{\perp}$, then based on ref. [22] the $D_{F}$ and $D_{S}$ for an axon with a $r(\theta, \varphi)$ orientation expressed in spherical coordinates $(\theta, \varphi)$, and where $I$ is the identity matrix, are:

$$
\begin{aligned}
& D_{F}(\theta, \varphi)=\left[\hat{r}(\theta, \varphi) \hat{r}(\theta, \varphi)^{T}\right] \lambda_{\|} \\
& D_{S}(\theta, \varphi)=\left[I-\hat{r}(\theta, \varphi) \hat{r}(\theta, \varphi)^{T}\right] \lambda_{\perp}
\end{aligned}
$$

The $\lambda_{\|}$corresponds to diffusion parallel to the axon, and $\lambda_{\perp}$ to diffusion perpendicular to the axon, and they were both collected from Table 1 in ref. [14]; corresponding, respectively, to the $D_{F}$ and $D_{S}$ experimental averages in that ref. [22] table.

If we consider $\vec{q}$ as the q-vector given by the product of the proton gyromagnetic ratio $\gamma$, with the diffusion-sensitizing gradient duration $\delta$, and the magnetic field gradient vector $\vec{g}$ [18], $\hat{q}$ as the unit-size q-vector, $\tau$ as the regularized diffusion-time, and $\zeta$ as a scalar between 0 and 1 (from Table 1 in ref. [14]), then the dMRI signal reduction for an orientation $(\theta, \varphi)$ is:

$$
S(\theta, \varphi) \vec{q}=\xi e^{-|\vec{q}|^{2} \tau \hat{q}^{\mathrm{T}} D_{S}(\theta, \varphi) \hat{q}}+(1-\xi) e^{-|\vec{q}|^{2} \tau \hat{q}^{\mathrm{T}} D_{F}(\theta, \varphi) \hat{q}}
$$

The q-vector has units of one over length, and that length expresses the diffusion spatial length scale-order the dMRI signal is probing. We model this dMRI signal reduction starting from the ODF of the WM fiber orientations, $h(\theta, \varphi)$.
The WM fiber orientation ODF, $h$, is expressed as the sum of an isotropic and an anisotropic component, the first of which is given by a sphere of radius $R$, and the second by a number $k$ of Gaussian-like peaks indexed by $j$ and with standard deviation $\sigma_{j}$.

The theoretical dMRI signal eq. $E_{T}$ is obtained using Equations (1) and (2) on Equation (3) to define $S$, and then using the defined $h$ to obtain:

$E_{T}(\vec{q})=\frac{\int_{0}^{2 \pi} \int_{0}^{\pi} h(\theta, \varphi) S(\theta, \varphi)_{\vec{q}} \sin \theta d \theta d \varphi}{\int_{0}^{2 \pi} \int_{0}^{\pi} h(\theta, \varphi) \sin \theta d \theta d \varphi}$

If the experimentally obtained dMRI signal intensity is $E_{\text {exp }}$, then the difference $G_{D S I}$ between the experimental and theoretical dMRI signal for DSI is obtained by the sum, over all the $\vec{q}$ used in DSI, of the squared differences between $E_{\text {exp }}$, and $E_{T}$.

The $E_{T}$ was compared to $E_{\text {exp }}$, and the parameters that give the best approximation were selected by choosing the $R, L_{j}$, and $\sigma_{j}$ parameters that minimize the difference between $E_{T}$ and $E_{\text {exp }}$ for the used data acquisition method, and they so define the $h_{f i t}$ for that acquisition method. The parameter combinations are described in Table 1 and are based on diffusion parameters from ref. [22], all parameter combinations were assessed for each voxel (Fig. 3 is the example for one voxel).

The isotropic and anisotropic fractions were obtained from the volume obtained from the $h_{f i t}$. The total volume is calculated by:

$V_{\text {total }}=\int_{0}^{2 \pi} \int_{0}^{\pi} h_{f i t}(\theta, \varphi) \sin \theta d \theta d \varphi$

The isotropic volume corresponds to the volume of the sphere with radius equal to the minimum value of $h_{f i t}(\theta, \varphi)$; therefore $h_{f i t}(\theta, \varphi)$ in Equation (5) is replaced by the minimum of $h_{f i t}$ :

$V_{i s o}-\int_{0}^{2 \pi} \int_{0}^{\pi} \min \left(h_{f i t}\right) \sin \theta d \theta d \varphi$ 
Fig. 2 Example of DSI connections from one seed with colors depend on orientation. The lines are 1 -axis connections $(k=1)$, planes are 2-axis connections $(k=2)$, and crossing planes are 3 -axis connections $(k=3)$

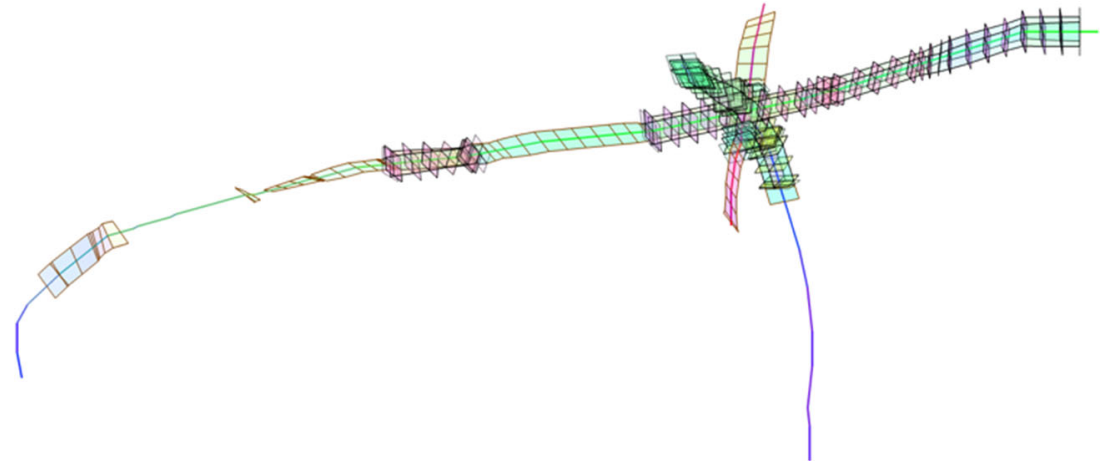

The anisotropic volume is the difference between total volume and isotropic volume: $V_{\text {aniso }}=V_{\text {total }}-V_{\text {iso }}$ The ratio between the anisotropic fraction of WM fibers, and the total amount of WM fibers is: $\Xi_{\text {aniso }}=\frac{V_{\text {aniso }}}{V_{\text {total }}}$. The ratio for the isotropic fractions (only calculated for WM voxels) is thus:

$\Xi_{\text {iso }}=\frac{V_{\text {iso }}}{V_{\text {total }}}$

\section{Processing of dMRI data}

The $\Xi_{i s o}$ of each voxel was correlated with the $k$ values obtained using the approach described in the next section. The study was performed on high quality DSI dMRI data from the Human Connectome Project (HCP). Henceforth, we will refer to these data as "HCP data". The HCP data used in the preparation of this work were obtained from the database of the MGH-UCLA section of the HCP (all the HCP data has been approved by the corresponding ethical committees and internal review boards) [23], the HCP data includes an anatomical T1-weigthed volume.

The HCP data had been processed for motion correction, using interspersed $b=0$ volumes every 20 volumes, and eddycurrent distortion correction, using the reversed-polarity method. The segmentation of the WM was performed in HCP data using FSL [24]. The anatomical data, and segmented maps were coregistered to diffusion space using FSL. Furthermore, we also used anatomical labeling provided by FSL; so that WM can be distinguished from gray matter (GM), from cerebro-spinal-fluid (CSF), and from everything else that is not WM. The anatomical image had its intensity inhomogeneity corrected, contrast adjusted, voxel re-sampled, and co-registered to the dMRI data. The values of TR and TE differed depending on which gradient orientation and amplitude is considered.

The HCP DSI data was acquired in Siemens 3 T scanners in a cubic q-space grid format, with 514 gradient directions, $2 \mathrm{~mm}$ isovoxels, an image size of $104 \times 104$, and 55 transversal slices. There were available only 2 subjects with data suitable for our analysis, for our analysis required data which was acquired using the maximum gradient strength and that could be processed using DSI approach. The subject 1, was acquired using a maximum gradient of $300 \mathrm{mT} / \mathrm{m}$, b maximum of $15,000 \mathrm{~s} / \mathrm{mm}^{2}$; whereas subject 2 was acquired at a maximum gradient of $90 \mathrm{mT} / \mathrm{m}$ and b-value maximum of $10,000 \mathrm{~s} / \mathrm{mm}^{2}$.

\section{Results}

The Diffusion Toolkit/TrackVis software [13] was used to obtain a 181 points ODF surface (not the axonal ODF). The obtained ODF data contains ODF peaks, which are the orientations for which the ODF is higher. The ODF peaks data binary data takes the value of 1 if the value of the ODF is a local maximum, and zero otherwise. For each peak, there is a peak pointing in the opposite direction with almost equal amplitude. If the ODF contains more than 3 pairs of opposing peaks, only the 3 highest ODF pairs of opposing peaks will be used. We call the orientation of a pair of ODF opposing peaks, an axis. In an axis, a connection to a neighboring voxel can be made by either advancing or retreating along that axis. We developed an automatic approach method based on previous works [15], which is a WM axis extension approach where for each WM point it is determined the number of axes parallel to the axis of another WM point. The obtained topological structure is a 3-plane crossing grid, such as occur in Fig. 2.

The number of coincident axes between two neighboring points is denoted by a non-negative integer $k$, implying that in

Table 1 The parameters used in our search

\begin{tabular}{lllllllllll}
\hline Parameters & $R$ & $L_{1}$ & $L_{2}$ & $L_{3}$ & $\sigma_{1}$ & $\sigma_{2}$ & $\sigma_{3}$ & $\lambda_{\|}$ & $\lambda_{\perp}$ & $\zeta$ \\
\hline Values & 1 & $0: 50: 300$ & $0: 50: 300$ & $0: 50: 300$ & $0: 0.1: 0.5$ & $0: 0.1: 0.5$ & $0: 0.1: 0.5$ & $1.69 \times 10^{-9}$ & $0.36 \times 10^{-9}$ & $20.91 \times 10^{-2}$ \\
\hline
\end{tabular}


Fig. 3 Experimental (blue line) and theoretical signals (green line) along the corresponding qvector number. The example voxel is on the position pointed by the green arrow near the ventricles (black triangular shapes) in the brain image overlaid by the isotropic fractions (yellow-red scale)

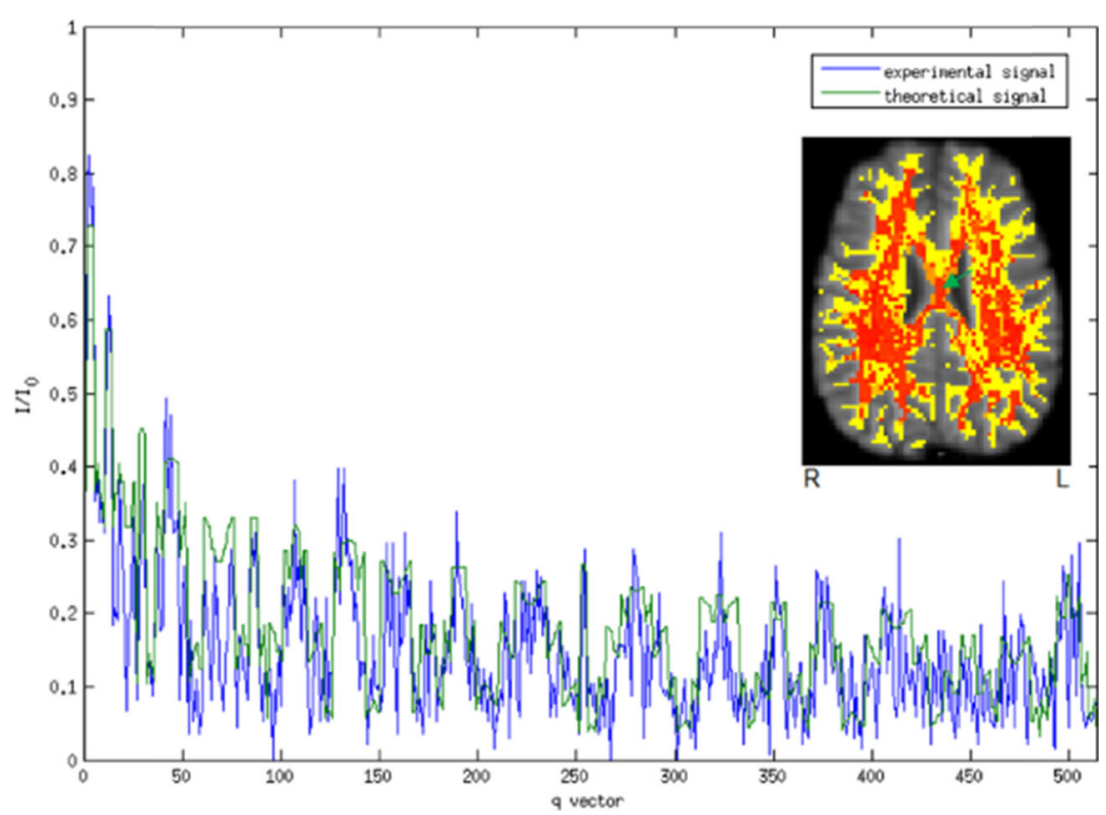

the same voxel there can be different $k$ values depending on which neighboring WM points are considered. The value of $k$ between two neighboring points defines the number of parallel fiber axes between the two sets of 3 axes, one set per point. The axes extension and parallelism detection are only performed for WM voxels, Fig. 4.

The isotropic fractions are higher near the vicinity of GM, as it is expected, since the fibers theoretically become less organized and with a less defined main direction. The regions with lower $\Xi_{\text {iso }}$ are mostly located in the Corpus Callosum (CC), superior longitudinal fasciculus, and corticospinal tracts (Fig. 4). The regions with lower isotropic fraction are in agreement with bigger $k$ values from the tractography method. It is apparent that high $k$ values correspond to low values of $\Xi_{\text {iso }}$. This was confirmed by calculating the mean values of the $\Xi_{\text {iso }}$ for each group of voxels with a given $k$ value, Table 2 . To assess the required amount of data needed to get the correct results without needing to deal with the multiple comparison problems, we used the Monte Carlo permutation test. The data were randomly sampled without replacement, so as to guarantee that the same data point is not sampled twice, This also limited the number of possible samples to the maximum number of data points existing with the feature of the population being sampled (e.g. $\mathrm{k}=1$ ). One of the advantages of the

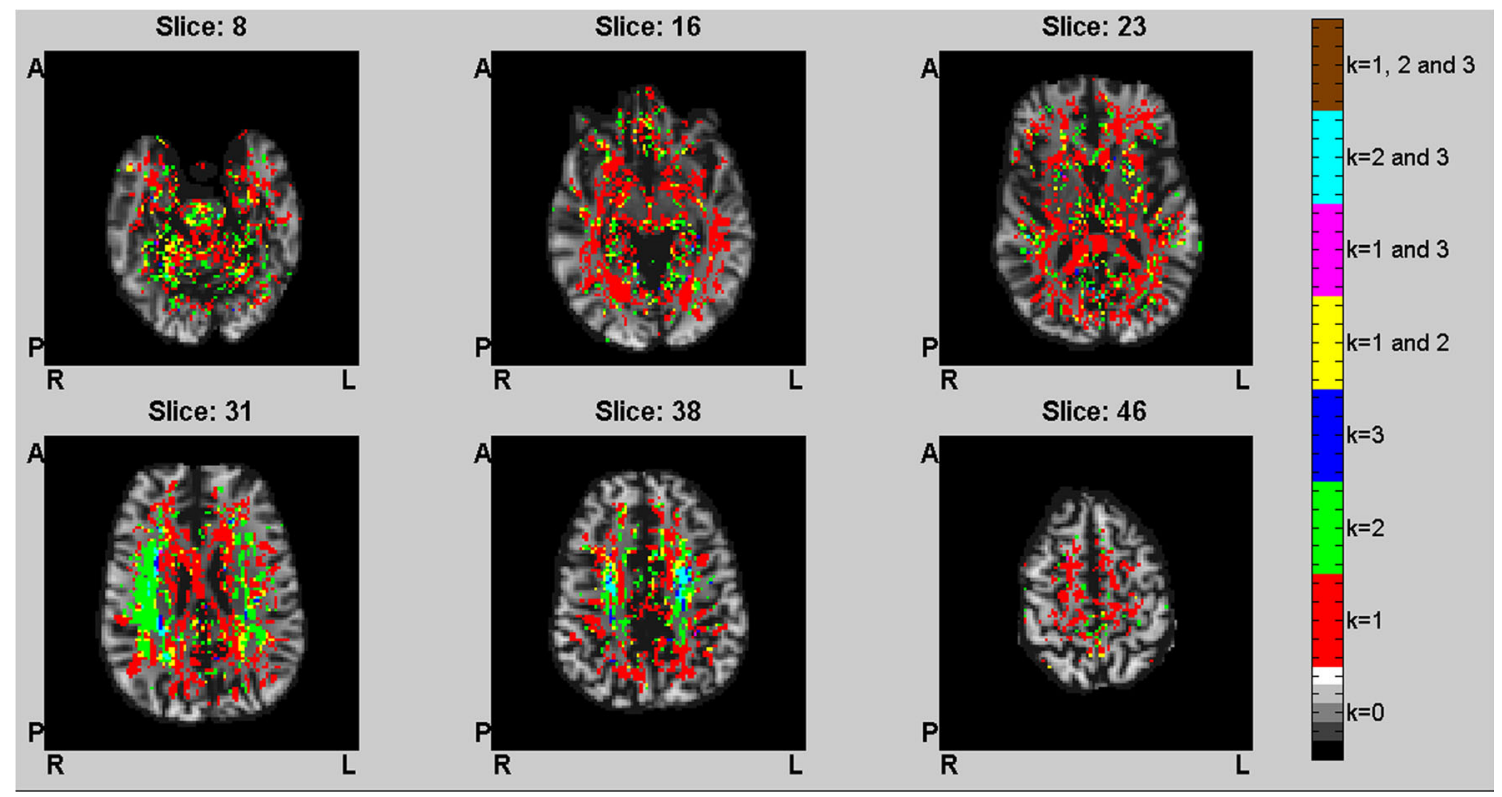

Fig. 4 Example of results using HCP DSI human data overlaid on HCP anatomical MRI 
Table 2 Isotropic fractions' $\Xi_{i s o}$ relation to $k$ values for two subjects. Only $k$ values with more than 5 voxels were considered statistically significant

\begin{tabular}{|c|c|c|c|c|c|c|c|c|}
\hline & & $\mathrm{k}=1$ & $\mathrm{k}=2$ & $\mathrm{k}=3$ & $\mathrm{k}=1 \mathrm{~V} 2$ & $\mathrm{k}=1 \mathrm{~V} 3$ & $\mathrm{k}=2 \mathrm{~V} 3$ & $\mathrm{k}=1 \mathrm{~V} 2 \mathrm{~V} 3$ \\
\hline \multirow[t]{2}{*}{ Subject 1} & $\left\langle\Xi_{i s o}\right\rangle \pm S E M$ & $63.1 \pm 0.5$ & $41.3 \pm 0.8$ & $38.7 \pm 1.8$ & $43.0 \pm 1.4$ & $53.7 \pm 6.2$ & $35.9 \pm 2.4$ & N/A \\
\hline & Number of voxels & 5777 & 2322 & 247 & 708 & 27 & 124 & 4 \\
\hline \multirow[t]{2}{*}{ Subject 2} & $\left\langle\Xi_{i s o}\right\rangle \pm S E M$ & $53.6 \pm 0.4$ & $37.4 \pm 0.7$ & $38.4 \pm 2.0$ & $38.8 \pm 1.3$ & $46.0 \pm 7.0$ & $33.0 \pm 2.4$ & $47.1 \pm 9.8$ \\
\hline & Number of voxels & 8251 & 3342 & 280 & 867 & 29 & 146 & 12 \\
\hline
\end{tabular}

Monte-Carlo method is that the sampling ordering is random, which reduces the systematic errors caused by sequential sampling.

The relation between the percentage of voxels used (x-axis) and the value of $\Xi_{\text {iso }}$ represented as a fraction of the $\Xi_{\text {iso }}$ obtained when all voxels are used (y-axis) appears in Fig. 5. We obtain the relations between the percentage of voxels used ( $\mathrm{x}$ axis) and the percentage of sub-partitions whose averages are
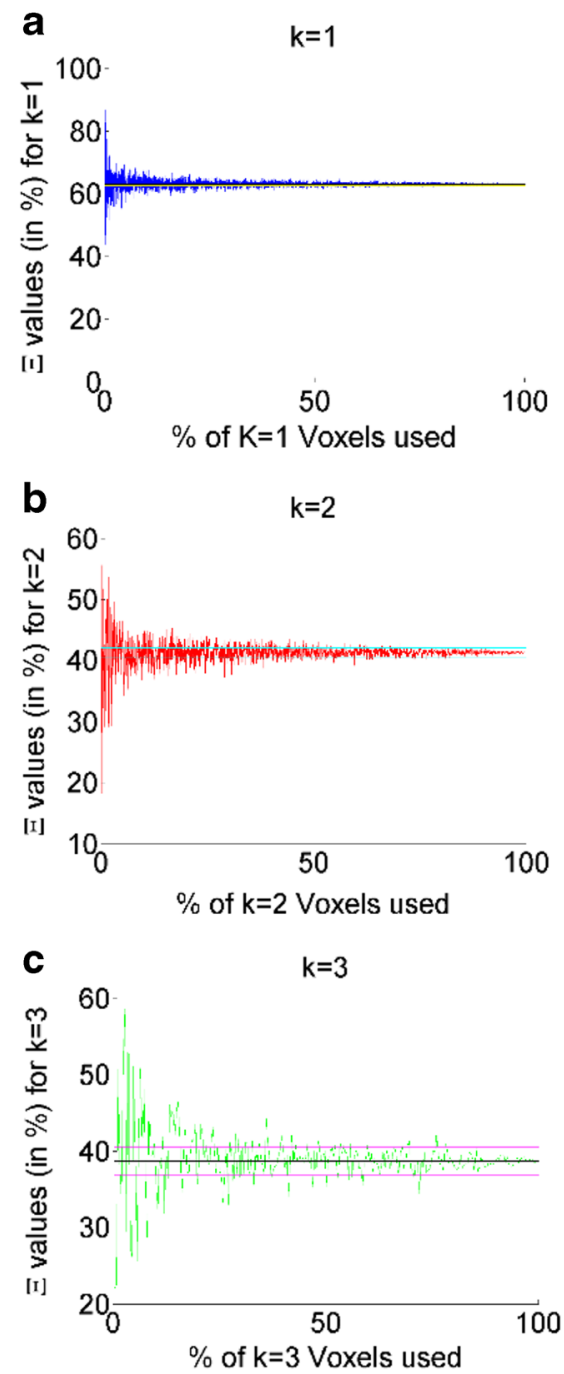

Fig. 5 Comparison of $\Xi_{i s o}$ calculated with all the voxels (straight line \pm 1 SEM) versus $\Xi_{\text {iso }}$ calculated with a Monte Carlo fraction of the voxels (y-axis) as a function of the fraction of the Monte Carlo-selected voxels (x-axis): $\mathbf{a} k=1, \mathbf{b} k=2, \mathbf{c}) k=3$ within \pm 1 standard error of the mean (SEM) of the true average (y-axis) (Fig. 6), the needed percentage of used voxels being defined when the percentage of means within \pm 1 SEM is $68 \%$, as it should be. These results were obtained for each of the three axis connections possibilities, specifically, $k=1, k=2$, and $k=3$. For both Fig. 5 and Fig. 6 the voxels are randomly sampled without replacement using a Monte Carlo method.
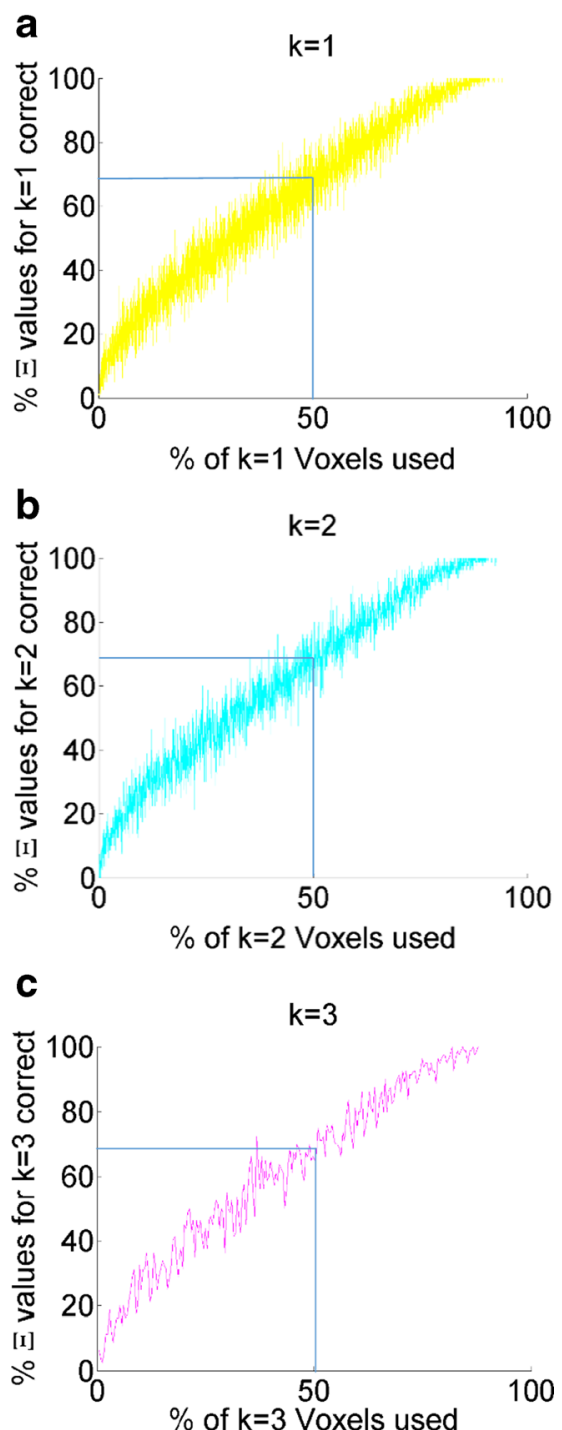

Fig. 6 Calculation using Monte Carlo method of the fraction of voxels with $\Xi_{\text {iso }}$ within \pm 1 SEM of the $\Xi_{\text {iso }}$ calculated with all the voxels (y-axis) as a function of the fraction of the Monte Carlo-selected voxels (x-axis): a $k=1, \mathbf{b} k=2, \mathbf{c}) k=3$ 


\section{Discussion}

Recent developments of new brain network technologies will soon allow for scalable data analysis. In diffusion data, high image resolutions are needed to accurately reconstruct connections, making the size of the input set very large [1]. Thus, time reduction in processing speed will have an exponential effect in big data platforms [1,6-8], especially in the context of current collaborative multicenter studies and also in clinical settings.

Here, we have shown a simple, powerful and high-quality method for time reduction when processing axonal structures of the brain. The high processing time required for the search of the model parameters limited the search of more optimal parameters, e.g. larger variations of $\lambda_{\|}$and $\lambda_{\perp}$. Our model is simple, but it provides a good approximation to the real axonal structures in the brain.

We found that the regions with higher $k$ values correspond to regions with lower $\Xi_{i s o}$. An arrangement $k=3$ between voxels suggests a lower isotropic fraction, which means that the 3-axis arrangement of the axons is a good representation of the majority of the axons' distribution, in agreement with ref. [15]. The simpler the arrangement, respectively $k=2$ and $k=1$, the higher the isotropic component of axonal distribution.

The neuroscience of big data has challenged the processing speed requirements, especially in the field of connectomics [1, 7]. Advances in brain network science produced an exponential increase in the need of computational power and time. Thus, urgent development of processing speed reduction methods for practical use are required [7]. The maximization of the information about structural brain connection needed for high quality results calls for new approaches for time reduction tools.

The alteration of brain connectivity is observed across a large range of psychiatric and neurological disorders. In fact, most of them have some degree of aberrant connectivity [2-5]. This knowledge will soon have direct applications for both clarification of the biology of these diseases as well as in drug discovery process. In the near future, it is very likely that pathoconnectomics will become part of the routine clinical assessment. In these setting, short time windows between recordings and evaluation became crucial.

Results provided here need to be replicated in larger samples, in different recordings with dissimilar scan qualities, and also in diverse neuropsychiatric conditions. Crucially, the application of this method to big data and multicenter recordings will determine the reliability, viability and impact of this method for massive data analysis.

We show here that the use of Monte Carlo voxel sampling obtained the correct result within 1 standard error of the mean (SEM) for $100 \%$ of the trials, using only about $50 \%$ of the data. It is this reduction in $50 \%$ of the data needed to obtain a correct enough result, that allows us to say that the Monte Carlo method is capable of reducing the computation time by about $50 \%$ without any reduction in result quality.

\section{Conclusion}

The brain structural connections among regions and their mapping to clinical and individual differences in healthy, psychiatric and neurological domains will have a critical growing with future big data connectome, open science resources, and massive clinical assessment [6]. Here we show that the use of Monte Carlo voxel sampling obtained the correct result within \pm 1 standard (SEM) $68 \%$ of the times using only about $50 \%$ of the data, which is what allows us to say that the Monte Carlo method is capable of reducing the computation time by about $50 \%$ without loss in result quality. This result opens a new agenda for the potential development of time-reduction methods in structural brain networks.

Acknowledgments We thank the financial support by QREN, FEDER, COMPETE, Investigador FCT, FCT Ciencia 2007, FCT PTDC/SAUBEB/100147/2008, FCT Project Scope UID/CEC/00319/2013, and the ERASMUS projects (FCT stands for "Fundação para a Ciência e Tecnologia"). We are thankful the relevant scientific conversations with Alard Roebroeck, Rainer Goebel, Van Wedeen, and Gina Caetano. Data collection for this work was in part from "Human Connectome Project" (HCP; Principal Investigators: Bruce Rosen, M.D., Ph.D., Arthur W. Toga, Ph.D., Van J. Weeden, MD). HCP funding was provided by the National Institute of Dental and Craniofacial Research (NIDCR), the National Institute of Mental Health (NIMH), and the National Institute of Neurological Disorders and Stroke (NINDS). HCP data are disseminated by the Laboratory of Neuro Imaging at the University of Southern California.

\section{References}

1. Lichtman, J.W., Pfister, H., and Shavit, N., The big data challenges of connectomics. Nat. Neurosci. 17(11):1448-1454, 2014.

2. Barkhof, F., Haller, S., and Rombouts, S.A., Resting-state functional MR imaging: A new window to the brain. Radiology. 272(1):2949, 2014.

3. Worbe, Y., Neuroimaging signature of neuropsychiatric disorders. Curr. Opin. Neurol. 28(4):358-364, 2015.

4. Zhou, J., and Seeley, W.W., Network dysfunction in Alzheimer's disease and frontotemporal dementia: Implications for psychiatry. Biol. Psychiatry. 75(7):565-573, 2014.

5. Sharp, D.J., Scott, G., and Leech, R., Network dysfunction after traumatic brain injury. Nat. Rev. Neurol. 10(3):156-166, 2014.

6. Craddock, R.C., Tungaraza, R.L., and Milham, M.P., Connectomics and new approaches for analyzing human brain functional connectivity. Gigascience. 4:13, 2015.

7. Marder, E., Understanding brains: details, intuition, and big data. PLoS Biol. 13(5):e1002147, 2015.

8. Boubela, R.N., et al., Big data approaches for the analysis of largescale fMRI data using apache spark and GPU processing: A demonstration on resting-state fMRI data from the human connectome project. Front Neurosci. 9:492, 2015. 
9. Conturo, T.E., Lori, N.F., Cull, T.S., Akbudak, E., Snyder, A.Z., et al., Tracking neuronal fiber pathways in the living human brain. Proc. Natl. Acad. Sci. U. S. A. 96:10422-10427, 1999.

10. Lori, N.F., Akbudak, E., Shimony, J.S., Cull, T.S., Snyder, A.Z., et al., Diffusion tensor fiber tracking of human brain connectivity: aquisition methods, reliability analysis and biological results. $N M R$ Biomed. 15:494-515, 2002.

11. Tuch, D.S., Q-ball imaging. Magn. Reson. Med. 52:1358-1372, 2004.

12. Behrens, T.E.J., Berg, H.J., Jbabdi, S., Rushworth, M.F.S., and Woolrich, M.W., Probabilistic diffusion tractography with multiple fibre orientations: What can we gain? Neuroimage. 34:144-155, 2007.

13. Wedeen, V.J., Wang, R.P., Schmahmann, J.D., Benner, T., Tseng, W.Y.I., et al., Diffusion spectrum magnetic resonance imaging (DSI) tractography of crossing fibers. Neuroimage. 41:12671277, 2008.

14. Raffelt, D., Tournier, J.D., Rose, S., Ridgway, G.R., Henderson, R., et al., Apparent Fibre Density: A novel measure for the analysis of diffusion-weighted magnetic resonance images. Neuroimage. 59: 3976-3994, 2012.

15. Wedeen, V.J., Rosene, D.L., Wang, R., Dai, G., Mortazavi, F., et al., The geometric structure of the brain fiber pathways. Science. 335: 1628-1634, 2012.

16. Dani, A., Huang, B., Bergan, J., Dulac, C., and Zhuang, X., Superresolution imaging of chemical synapses in the brain. Neuron. 68:843-856, 2010
17. Hawrylycz, M.J., Lein, E.S., Guillozet-Bongaarts, A.L., Shen, E.H., Ng, L., et al., An anatomically comprehensive atlas of the adult human brain transcriptome. Nature. 489:391-399, 2012.

18. Tuch, D.S., Reese, T.G., Wiegell, M.R., and Wedeen, V.J., DMRI of complex neural architecture. Neuron. 40:885-895, 2003.

19. Hill, S.L., Wang, Y., Riachi, I., Schürmann, F., and Markram, H., Statistical connectivity provides a sufficient foundation for specific functional connectivity in neocortical neural microcircuits. Proc. Natl. Acad. Sci. U. S. A. 109:E2885-E2894, 2012.

20. Wang, R., Benner, T., Sorensen, A.G., and Wedeen, V.J., Diffusion toolkit: A software package for diffusion imaging data processing and tractography. Proc. Intl. Soc. Mag. Reson. Med. 15:3720, 2007.

21. Assaf, Y., Blumenfeld-Katzir, T., Yovel, Y., and Basser, P.J., AxCaliber: a method for measuring axon diameter distribution from dMRI. Magn. Reson. Med. 59:1347-1354, 2008.

22. Milne, M.L., and Conradi, M.S., Multi-exponential signal decay from diffusion in a single compartment. J. Magn. Reson. 197:8790, 2009.

23. U.C.L.A. (n.d.) LONI Image Data Archive (IDA). Available: https://ida.loni.ucla.edu/login.jsp. Accessed 16 November 2012. (2012)

24. Zhang, Y., Brady, M., and Smith, S., Segmentation of brain MR images through a hidden Markov random field model and the expectation-maximization algorithm. IEEE Trans. Med. Imaging. 20:45-57, 2001. 